\title{
Design of Train Speed Controller Based on Generalized Predictive Control
}

\author{
Jiaxi Yuan ${ }^{1}$, Xiangxian Chen ${ }^{1}$, Chenge Geng ${ }^{1}$, Zhujun Ling ${ }^{2}$, Dandan Liu ${ }^{2}$, and Guantao Hu ${ }^{2}$ \\ ${ }^{1}$ Department of Instrumentation Science and Engineering, Zhejiang University, Hangzhou, China \\ ${ }^{2}$ Research and Development Center, Zhejiang United Science and Technology Co., Ltd, Hangzhou, China
}

\begin{abstract}
In order to realize the automatic control of train running process, a generalized predictive control based speed controller is designed. GPC is a kind of control algorithm which is easy to be implemented. Based on the GPC algorithm, the speed controller is combined with the stair-like control scheme, input constraints and predictive feedback in this paper. Through the ATO simulation system, the controller is proved to be useful and effective.
\end{abstract}

Keywords: automatic train operation, generalized predictive control, overshoot suppression, input constraints

\section{Introduction}

ATC (Automatic Train Control) system is the most important part of urban rail transit system. ATC system ensures the safety and efficiency of urban rail transit system. By means of modern technologies such as communication, computer and control, the functions of automatic operation, operation management, commanding and dispatching are realized [1]. ATO (Automatic Train Operation) system is a subsystem of ATC system, which is an important part to ensure the quality of train operation. ATO system has been widely used in the control of urban rail trains [2].

In the application of the ATO system, the most widely used algorithm is PID which is the representative of the classical control algorithm [3]. PID has advantages of easy realization and simple principles, but it is lack of flexibility. PID controller will increase the energy consumption in the process of traction and braking adjustment. And it is not conducive to the comfort of passengers. It cannot fully satisfy the growing demands.

GPC (Generalized Predictive Control) [4] is a kind of control algorithm based on prediction model. Through the prediction model, online calculation, rolling optimization and feedback correction, the system can achieve the target. Based on the GPC algorithm, the speed controller designed in this paper is combined with the stair-like control scheme, input constraints and predictive feedback. The effectiveness of the controller is verified by the ATO simulation system.

\section{Automatic train Operation System}

The main function of ATO system is to operate the train in place of the experienced driver. ATO system can automatically adjust the running speed of the train and park the train smoothly. Besides, the ATO system can improve the operation qualities such as efficiency and comfort. The tasks of ATO system rely on the coordination of other subsystems of ATC system [5]. According to the real-time information such as speed limit information, line parameters, speed and location received from ATP system, ATO adjusts the running speed to adapt to the changing operation conditions. Fig. 1 shows the collaboration among the ATO system, ATP system and ATS system.

\footnotetext{
${ }^{1}$ Corresponding author. Tel.: + 8615957187314 .

E-mail address: sherryueda@126.com.
} 
The train is the controlled object of the ATO system and the control input information are the traction command and the braking command. The simplified process of adjusting the train speed by ATO system can be presented as Fig 2 . Ref $(\mathrm{k})$ is the train target speed, $\mathrm{u}(\mathrm{k})$ is the control commands and $\mathrm{y}(\mathrm{k})$ is the actual train speed.

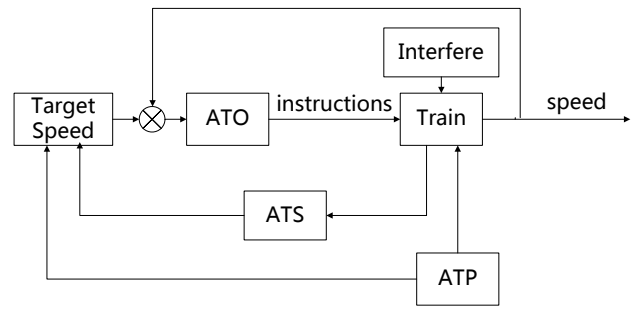

Fig. 1: Collaboration between ATO, ATP and ATS systems

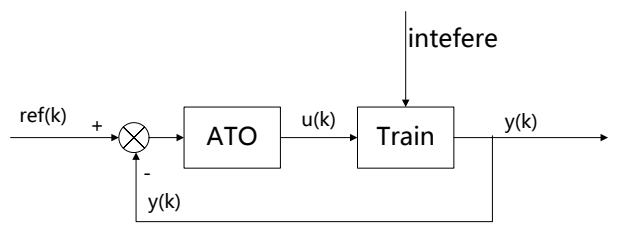

Fig. 2: Simplified Process of ATO

\section{Design and Implementation of ATO Simulation System}

\subsection{Model of the controlled train}

For the ATO system, it is necessary to establish a train model as the controlled object. Generally, the slope of the train track is small, and the radius of the bend is much larger than the length of the train. The train model based on single particle model can be established according to Newton's law. The resultant force of the train is composed of traction/braking force and resistance.

$$
\left\{\begin{array}{l}
d v / d t=c F \\
F=u-w \\
w=\alpha_{0}+\alpha_{1} v+\alpha_{2} v^{2}
\end{array}\right.
$$

where, $t$ is time, $v$ is the speed, $c$ is the acceleration coefficient, $F$ is the resultant force of train, $u$ is the traction/braking force, $w$ is the resistance; $\alpha_{0}, \alpha_{1}, \alpha_{2}$ are the resistance coefficients.

The train model of formula (1) is represented by the following discrete model.

$$
y(k)=\boldsymbol{\varphi}^{\mathrm{T}}(k) \boldsymbol{\theta}(k)+\delta(k)
$$

where, the definition of the parameter vector is $\boldsymbol{\theta}(k)$ and the information vector is $\boldsymbol{\varphi}(k)$.

$$
\begin{gathered}
\boldsymbol{\theta}(k)=\left[a_{1}(k), a_{2}(k), b_{1}(k), d_{0}(k)\right]^{\mathrm{T}} \\
\boldsymbol{\varphi}(k)=\left[y(k-1)^{2}, y(k-1), u(k-1), 1\right]^{\mathrm{T}}
\end{gathered}
$$

where, $y$ is the speed; $u$ is the traction/braking force; $\delta(k)$ is the random noise; $a_{1}(k), a_{2}(k), b_{1}(k), d_{0}(k)$ are the parameters of the model.

\subsection{Target speed}

In the process of operation, the train needs to change the operating modes according to the external environment. In the same environment, the different operating modes of the train will result in different operation time and energy consumption. In order to keep the train running in high performance, it is necessary to arrange the operating modes of the train reasonably.

The train operating modes can be divided into three kinds: traction, coasting and braking modes. There are corresponding conversion rules between different operating modes. The train can change from coasting mode to traction or braking mode directly. But there must be the coasting mode between traction mode and braking mode.

The overall target curve uses the traction-coasting-braking transformation mode. 
Traction part uses quadratic function, the formula is as follows:

$$
v=\left\{\begin{array}{l}
a\left(t-t_{0}\right)^{2}+v_{0}, t_{0} \leq t \leq t_{1} \\
-a\left(t-t_{2}\right)^{2}+v_{2}, t_{1} \leq t \leq t_{2}
\end{array}\right.
$$

Braking part uses quadratic function, the formula is as follows:

$$
v=\left\{\begin{array}{l}
-a\left(t-t_{2}\right)^{2}+v_{2}, t_{0} \leq t \leq t_{1} \\
a\left(t-t_{0}\right)^{2}+v_{0}, t_{1} \leq t \leq t_{2}
\end{array}\right.
$$

The final train target curve is shown below.

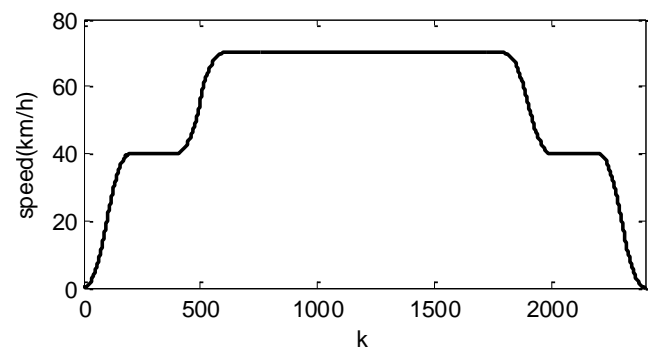

Fig. 3: Target Speed

\subsection{GPC based speed controller}

GPC (Generalized predictive control) is a kind of predictive control algorithm which has been widely used in industrial control. It has the characteristics of predictive model, rolling optimization and feedback correction [6]. The controlled object is described by CRIMA (Controlled Autoregressive Integrated Moving Average) model. Based on the GPC algorithm, the train speed controller in this paper is combined with the stair-like control scheme, input constraints and predictive feedback. The structure of the train speed controller is shown in Fig. 4.

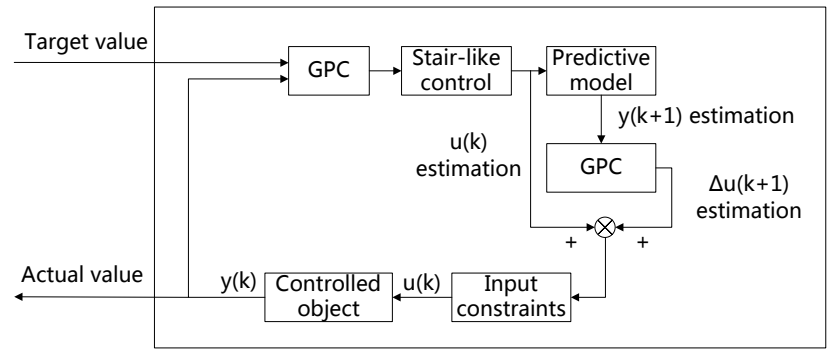

Fig. 4: Structure of the GPC Based Train Speed Controller

The target curve is used as the reference curve of train. According to the input and output data collected from the train, the VFF-RLS [7] method is used to update the prediction model in order to ensure the accuracy of the predicted value. The updated prediction model is used to estimate the multi step prediction which will be compared with the reference curve. The original GPC method and stair-like control scheme are combined to obtain the current optimal control input. According to the calculated optimal control input, the control input of the next moment can be estimated. And we can use the estimated value to make compensation to the input at current moment. Finally, according to the input constraints, the compensated control input is modified. The advanced speed controller can not only track the train speed, but also improve the control precision and the running stability.

\section{Simulation}

According to the train model and train control algorithm in this paper, the simulation experiment is carried out based on the running data collected from Hangzhou line 4. The advanced speed controller based on GPC is used to control the train. The effectiveness of the proposed controller is verified by the simulation results.

The simulation results of the speed are shown in the following figure. 


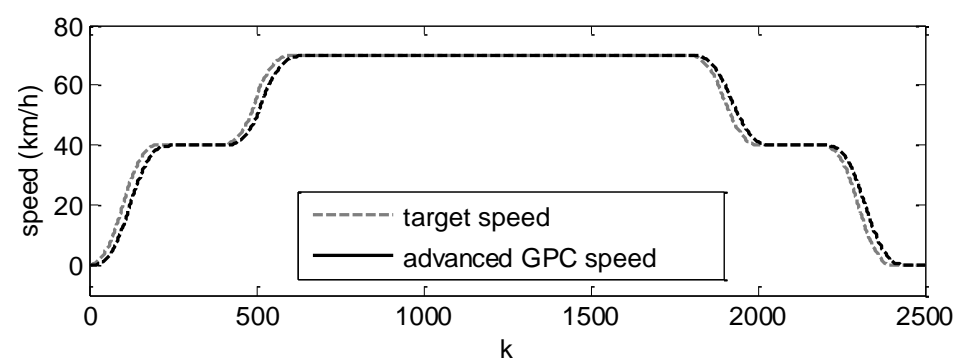

Fig. 5: Control result under the advanced GPC

As can be seen from Fig 5, the advanced GPC speed controller can track the target speed accurately and the controlled speed curve is quite smooth.

Under the same conditions, the original GPC speed controller is also used and the changes of the input increment are compared with the advanced one. The results are shown in Fig 6.

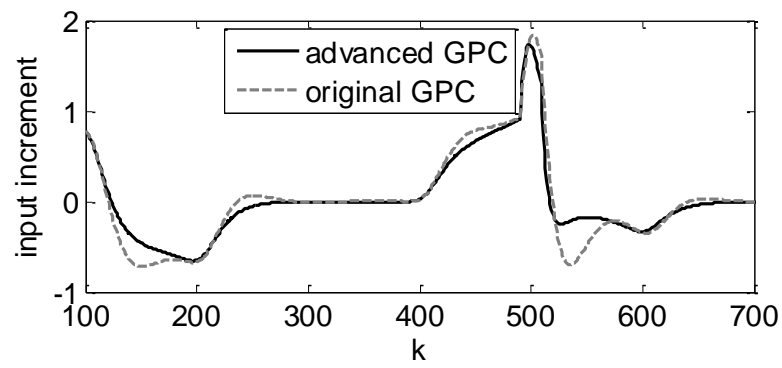

Fig. 6: Input Increment Results

Compared to the original GPC speed controller, the input increment of the advanced GPC speed controller is smaller, which embodies the advantages of the stair-like control. Thus, the train speed controller in this paper can reduce the cost of changing the traction/braking force in the control process.

When there are input constraints, the compared input results of the two controllers are shown in Fig 7.

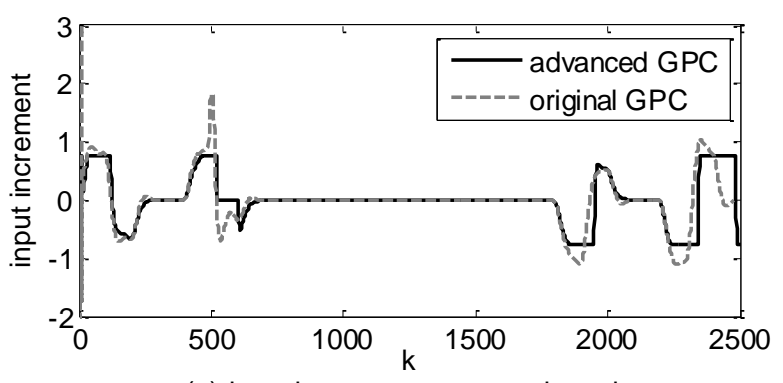

(a) input increment compared results

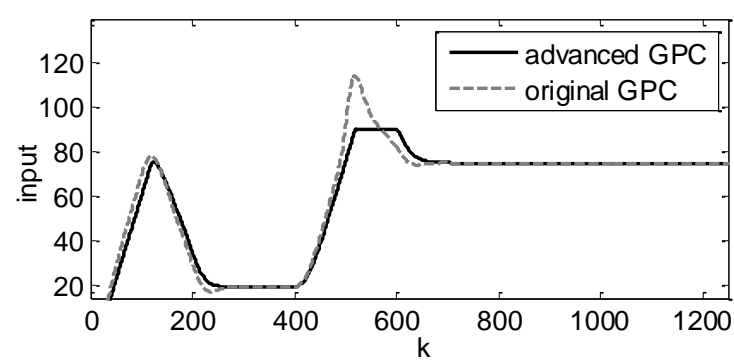

(b)input compared results

Fig. 7: Input Constraint Results

According to Fig. 7, it can be seen that the advanced GPC speed controller can restrain the input, so that the control input will not exceed the input constraints.

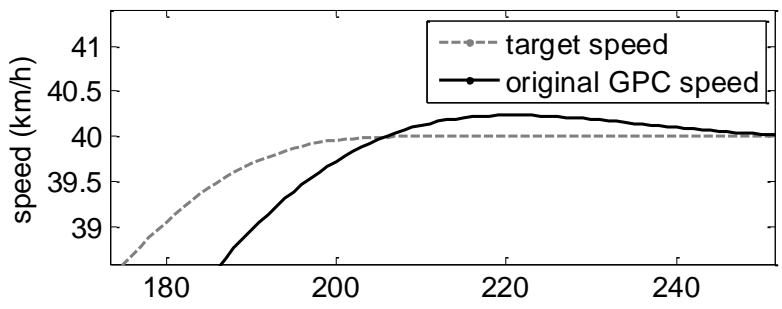

(a) overshoot of original GPC

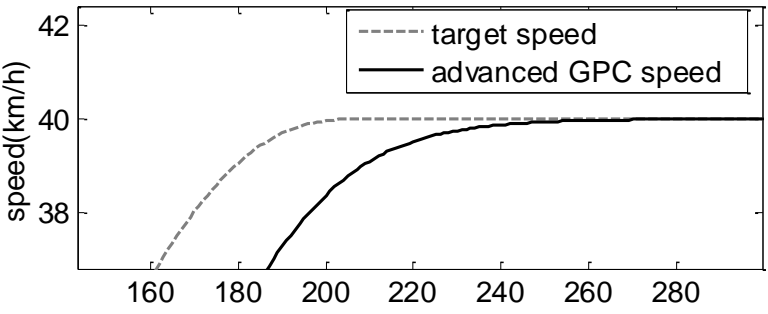

(b)overshoot of ${ }^{\mathrm{k}}$ advanced GPC

Fig. 8: Overshoot Suppress Results

Fig. 8 shows the comparison of the overshoot suppress results of the two controllers. 
If there is overshoot when the train is running, it may lead to over speed or even cause emergency stop, which will affect the safety of the train system. Fig. 8 shows that the controlled speed of the original GPC exceeds the target speed, while the overshoot can hardly be observed in the advanced one. The controller proposed in this paper has the advantage of suppressing overshoot.

The following figures show the control results of time-varying train model.

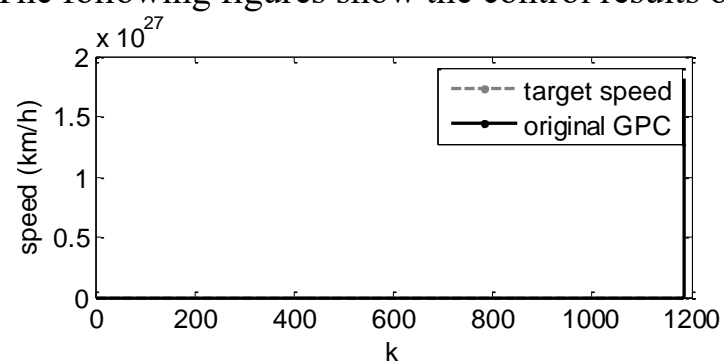

(a) original GPC

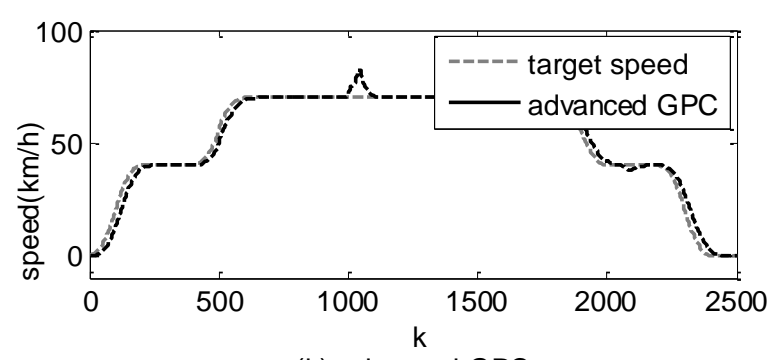

(b) advanced GPC

Fig. 9: time-varying system control results

When the train model changes with the environment, the original GPC has mutation problem due to the singular matrix inversion, and it cannot satisfy the control needs. Because of the stair-like control scheme, the advanced GPC can avoid the computation of the inverse matrix and can track the target speed in time.

\section{Conclusion}

In this paper, a GPC based ATO simulation system is designed and implemented. The GPC based controller is used to control the train speed and the results are compared with the original GPC controller. The effectiveness and superiority of the controller designed in this paper is verified. The stair-like control scheme is applied to avoid the matrix inverse operation. Besides, the future input are estimated and regarded as feedback in order to suppress the overshoot. At the same time, the input constraints are considered so that the controller can be applied in real ATO system. The simulation results show that the train speed controller designed in this paper can satisfy the needs of ATO system and it provides a basis and reference value for the application of predictive control in ATO system.

\section{Acknowledgements}

The authors wish to acknowledge Jing Zheng of Zhejiang University, who provided plenty of suggestions for this paper. This work is supported by the funding from the National Science and Technology Infrastructure Program of China under Grant 2015BAG19B03.

\section{References}

[1] C. J. Wan, "Design and implementation of automatic train control system", Journal of Xi' an Petroleum Institute (Natural Science Edition), 2005, vol. 18, pp. 69-72.

[2] H. D. Liu, B. H. Mao, Y. Ding and T. J. He, "A Study on Simulation Algorithm and Operation of Automatic Train Operation”, Journal of System Simulation, 2005, vol. 17, pp.577-580.

[3] T. Tao and L.J. Huang, "A Survey of Control Algorithm for Automatic Train Operation”, Journal of the China Railway Society, 2003, vol.25, pp.98-102.

[4] D. W. Clarke, C. Mohtadi, "Properties of Generalized Predictive Control", Automatica,1989, vol.25, pp. 859875.

[5] J. Y. Zhou and T. Tang, "Control Algorithm of Automatic Train Operation System", China Science and Technology Information, 2008, vol. 7, pp.306-309.

[6] Y. H. Hu and X. L. Jia, “ A Direct Generalized Predictive Control Algorithm”, Control and Decision, 2000, vol. 15, pp.221-223.

[7] Z. Li, Z. Young, H. Young and J. Liu, "Generalized Predictive Control with Dual Adaptation Method of High Speed Train”, China Railway Science, 2015, vol. 36, pp.120-127. 